\title{
Neonatal administration of tamoxifen causes disruption of myometrial development but not adenomyosis in the C57/BL6J mouse
}

\author{
Mohamed K Mehasseb, S C Bell and M A Habiba \\ Reproductive Sciences Section, Department of Cancer Studies and Molecular Medicine, University of Leicester, \\ Robert Kilpatrick Clinical Sciences Building, Leicester LE2 7LX, UK \\ Correspondence should be addressed to M K Mehasseb; Email: mkm7@le.ac.uk
}

\begin{abstract}
We previously demonstrated that in the CD-1 mouse, which exhibits a high incidence of age-related adenomyosis, neonatal exposure to tamoxifen induced premature uterine adenomyosis and was associated with abnormal development particularly of the inner myometrium. In the present study, we examined the effect of neonatal tamoxifen administration upon uterine development in the C57/BL6J mouse strain that is not known to develop uterine adenomyosis. Female C57/BL6J pups ( $n=20)$ were treated with oral tamoxifen ( $1 \mathrm{mg} / \mathrm{kg})$ from age 1 to 5 days. Uteri from control and treated mice were obtained on days $5,10,15$ and 42 of age. We examined sections histologically using image analysis and immunohistochemistry for $\alpha$-smooth muscle actin (ACTA2, $\alpha$-SMA), desmin, vimentin, laminin, fibronectin and oestrogen receptor- $\alpha$ (ESR1). Following tamoxifen exposure, all uteri showed inner myometrium thinning, lack of continuity, disorganisation and bundling. However, adenomyosis was not seen in any uterus. ACTA2 immunostaining was less in the circular muscle layer of treated mice. The temporal pattern of desmin immunostaining found in control mice was absent in tamoxifen-treated mice. There was no difference in the localisation of laminin or fibronectin between control and tamoxifen-treated groups. However, laminin immunostaining was reduced in the circular muscle layer of treated mice. Vimentin could not be detected in either group. In conclusion, our results demonstrate that the development of the inner myometrium is particularly sensitive to oestrogen antagonism, and is affected by steroid receptor modulation. Although tamoxifen induces inner myometrial changes including that of ACTA2, desmin, ESR1 and laminin expression in C57/BL6J neonatal mice similar to those induced in CD-1 mice, C57/BL6J mice did not develop premature adenomyosis. Thus, disruption of the development and differentiation of the inner myometrium cannot alone explain the development of tamoxifen-associated adenomyosis, and this must be dependent upon its interaction with strain-dependent factors. Reproduction (2010) 139 1067-1075
\end{abstract}

\section{Introduction}

Uterine adenomyosis is characterised by the presence of endometrial glands and/or stroma deep within a hypertrophic and hyperplastic myometrium (Bird et al. 1972). The pathophysiology of adenomyosis is poorly understood, but risk factors in women include age, parity, sharp curettage during early pregnancy and tamoxifen use (Levgur et al. 2000, Curtis et al. 2002). Elucidation of the mechanisms involved in its initiation and development in women is difficult, partly because diagnosis is rarely made except after hysterectomy. Development of an animal model will thus be useful for the study of the disease.

Spontaneously occurring adenomyosis has been reported in several animal species including nonhuman primates, dogs, cats, laboratory rodents and rabbits (McCann \& Myers 1970, Suire et al. 1978). In mice, its prevalence is strain dependent. In CD-1 mice, spontaneous adenomyosis appears from about 6 months of age. By 12 months of age, over $80 \%$ are affected, with disease severity increasing thereafter (Greaves \& White 2006). In contrast, the C57/BL6] mouse strain is not known to develop spontaneous adenomyosis and is resistant to experimental adenomyosis (Mori et al. 1982, 1991, Irisawa \& Iguchi 1990). Various forms of hormonal manipulation can enhance the development of adenomyosis in the mouse, although the effectiveness of this manipulation is also strain dependent (Mori et al. 1982). These include hypothalamic autotransplant-induced hyperprolactinaemia (Huseby \& Thurlow 1982, Mori \& Nagasawa 1983) and prolonged oestrogen (Guttner 1980) or progesterone administration (Ostrander et al. 1985).

More recently, neonatal oral administration of tamoxifen to female CD-1 pups has been demonstrated to induce the premature appearance of adenomyosis by 6 weeks of age rather than at 6 months in untreated 
animals (Parrott et al. 2001, Green et al. 2005). This effect was tamoxifen specific in that oestradiol $\left(\mathrm{OE}_{2}\right)$ or raloxifene failed to induce adenomyosis. In a recent detailed study, development of adenomyosis in CD-1 mice has been reported to occur as early as day 10 , with over $80 \%$ of uteri being affected by day 15 (Mehasseb et al. 2009). The earliest changes detected included discontinuity in the inner myometrial layer and ingrowth of endometrial glands and stroma. The inner circular muscle layer exhibited marked thinning, lack of continuity, and lack of orientation and bundling of the muscle cells. Desmin expression in these cells was markedly reduced. The findings suggested that abnormal development of the inner circular muscle layer resulting in its discontinuity may play a role in the development of premature uterine adenomyosis (Mehasseb et al. 2009). In the present study, we examined the effect of neonatal tamoxifen administration on the C57/BL6J mouse strain upon early uterine development.

\section{Results}

In the following subsections, we first present the uterine development in the control C57/BL6J mice.
Then, we describe the morphological changes observed in the tamoxifen group. We finally present our data on immunohistochemical markers. The data are visually presented and summarised in Tables 1 and 2, and Figs 1-5.

\section{Morphology and uterine development}

\section{Normal uterine development of C57/BL6J mice}

Day 5. The uterine cavity had an oval-shaped lumen elongated in the mesometrial-antimesometrial axis (Table 1). The luminal epithelium had tall columnar cells with elongated nuclei arranged in palisade with some multilayered areas. There was no evidence of gland formation. In the uterine wall, the mesenchymal cells started to segregate into three layers: endometrial stroma, inner circular and prospective outer longitudinal muscle layers. The prospective inner circular muscle layer was the most defined and densely staining. The cells were bundled, circularly orientated, and tightly packed with elongated nuclei and minimal intercellular space. Vascular spaces started to appear, especially in the layer between the inner and outer myometrium (Figs 1A-5A).

Table 1 Comparison of the histological features measured at different stages of development of neonatal C57/BL6J mice after administration of tamoxifen or vehicle only, with reference to the similar CD-1 model (Mehasseb et al. 2009). Measurements are in $\mu$ m: median (range).

\begin{tabular}{|c|c|c|c|c|}
\hline & \multicolumn{2}{|c|}{ C57/BL6J } & \multicolumn{2}{|c|}{ CD-1 (Mehasseb et al. 2009) } \\
\hline & Control & Tamoxifen & Control & Tamoxifen $^{\mathrm{a}}$ \\
\hline \multicolumn{5}{|l|}{ Day 5} \\
\hline Epithelial height & $10(8-12)$ & $12(10-12)$ & $18(16-20)$ & $25(25-35)$ \\
\hline Uterine wall thickness & $75(60-80)$ & $80(80-90)$ & $110(100-110)$ & $70(60-80)$ \\
\hline Inner cellular layer (future stroma) & $50(45-55)$ & $50(45-60)$ & $70(60-80)$ & $35(35-40)$ \\
\hline Inner circular muscle layer & $15(12-16)$ & $9(8-12)$ & $30(25-35)$ & $20(20-25)$ \\
\hline Outer cellular layer (future outer myometrium) & $10(8-12)$ & $10(8-13)$ & $12(10-15)$ & $12(10-15)$ \\
\hline \multicolumn{5}{|l|}{ Day 10} \\
\hline Epithelium height & $14(12-14)$ & $18(18-20)$ & $18(16-20)$ & $15(12-20)$ \\
\hline Uterine wall thickness & $100(90-110)$ & $110(90-115)$ & $120(110-130)$ & - \\
\hline Inner cellular layer (future stroma) & $55(50-60)$ & - & $90(80-100)$ & - \\
\hline Inner circular muscle layer & $25(20-26)$ & - & $30(25-35)$ & - \\
\hline Outer cellular layer (future outer myometrium) & $20(18-22)$ & - & $20(15-25)$ & - \\
\hline \multicolumn{5}{|l|}{ Day 15} \\
\hline Epithelial height & $12(12-14)$ & $14(13-15)$ & $18(15-20)$ & $18(15-20)$ \\
\hline Uterine wall thickness & $115(100-120)$ & $100(80-120)$ & $170(160-180)$ & - \\
\hline Endometrial stroma & $60(55-70)$ & - & $100(90-110)$ & - \\
\hline Inner circular myometrium & $25(22-28)$ & - & $40(35-40)$ & - \\
\hline Intermediate vascular layer & $10(8-12)$ & - & $10(10-15)$ & - \\
\hline Outer myometrium & $20(18-22)$ & $20(15-20)$ & $25(20-30)$ & $25(20-30)$ \\
\hline \multicolumn{5}{|l|}{ Day 42} \\
\hline Epithelial height & $14(12-16)$ & $20(15-20)$ & $18(15-20)$ & $20(15-20)$ \\
\hline Uterine wall thickness & $160(150-170)$ & $170(160-180)$ & $340(320-360)$ & - \\
\hline Endometrial stroma & $70(65-80)$ & - & $120(110-130)$ & - \\
\hline Inner circular muscle layer & $40(30-45)$ & - & $80(75-90)$ & - \\
\hline Intermediate vascular layer & $20(18-22)$ & - & $50(50-55)$ & - \\
\hline Outer myometrium & $30(28-34)$ & $35(30-50)$ & $90(85-100)$ & $50(40-60)$ \\
\hline
\end{tabular}

${ }^{a}$ The uteri in the CD-1 mice dosed with tamoxifen had wide irregular lumens and poorly defined layers characterised by penetrating stroma, markedly disrupted circular layer. The whole uterine structure was so disorganised with glands and surrounding stroma dispersed throughout the uterine wall, with few scattered muscle fibres and blood vessels. The wall thickness was measured from the luminal epithelium of the central lumen to the serosal edge. However, the central lumen was not identified in most CD-1 specimens treated with tamoxifen. Where the structures were intermingled and too indistinct to measure separately, some measurements were absent (-). 
Table 2 Immunohistochemical expression of desmin and vimentin in the uterus of C57/BL6J mice. All blood vessel walls stained strongly positive with vimentin.

\begin{tabular}{|c|c|c|c|c|}
\hline & \multicolumn{2}{|c|}{ Control } & \multicolumn{2}{|c|}{ Tamoxifen } \\
\hline & Desmin & Vimentin & Desmin & Vimentin \\
\hline \multicolumn{5}{|l|}{ Day 5} \\
\hline Inner layer & +++ & - & ++ & - \\
\hline Middle layer & ++ & - & + & - \\
\hline Outer layer & ++ & - & + & - \\
\hline \multicolumn{5}{|l|}{ Day 10} \\
\hline Stroma & ++ & - & + & - \\
\hline Inner myometrium & +++ & - & + & - \\
\hline Outer myometrium & ++ & - & + & - \\
\hline \multicolumn{5}{|l|}{ Day 15} \\
\hline Stroma & ++ & + & + & + \\
\hline Inner myometrium & ++ & - & + & - \\
\hline Outer myometrium & +++ & - & + & - \\
\hline \multicolumn{5}{|l|}{ Day 42} \\
\hline Stroma & + & + & + & + \\
\hline Inner myometrium & ++ & - & + & - \\
\hline Outer myometrium & +++ & - & + & - \\
\hline
\end{tabular}

Day 10. Uterine sections had a more complex luminal epithelium invaginating the stroma to form simple tubular uterine glands. All layers of the uterine wall were more distinct. The stroma appeared more tightly packed and surrounded the endometrial glands. The inner circular muscle layer was thicker compared to the outer myometrium, and cells were organised into bundles. Longitudinally cut sections demonstrated elongated nuclei in the outer layer, confirming its longitudinal arrangement. Blood vessels were larger and observed in all layers of the uterine wall (Figs 1B-5B).

Day 15. The adult configuration of the uterus was apparent. The endometrium had numerous simple tubular glands and a thick endometrial stroma. Stroma cells were randomly orientated, except around the individual glands where they appeared more circular. A distinct loose vascular layer separated the inner and outer myometrial layers. The outer myometrial cells became grouped in bundles connected by loose connective tissue sheaths and separated from the inner myometrium by a distinct loose vascular layer (Figs 1C-5C).

Day 42. The adult uterus was larger and more complex, but had the same configuration as the uterus on day 15 . The endometrial glands were more complex and branching, but were confined to the endometrial layer. The inner myometrium was quite distinct. The intervening vascular layer harboured most of the large blood

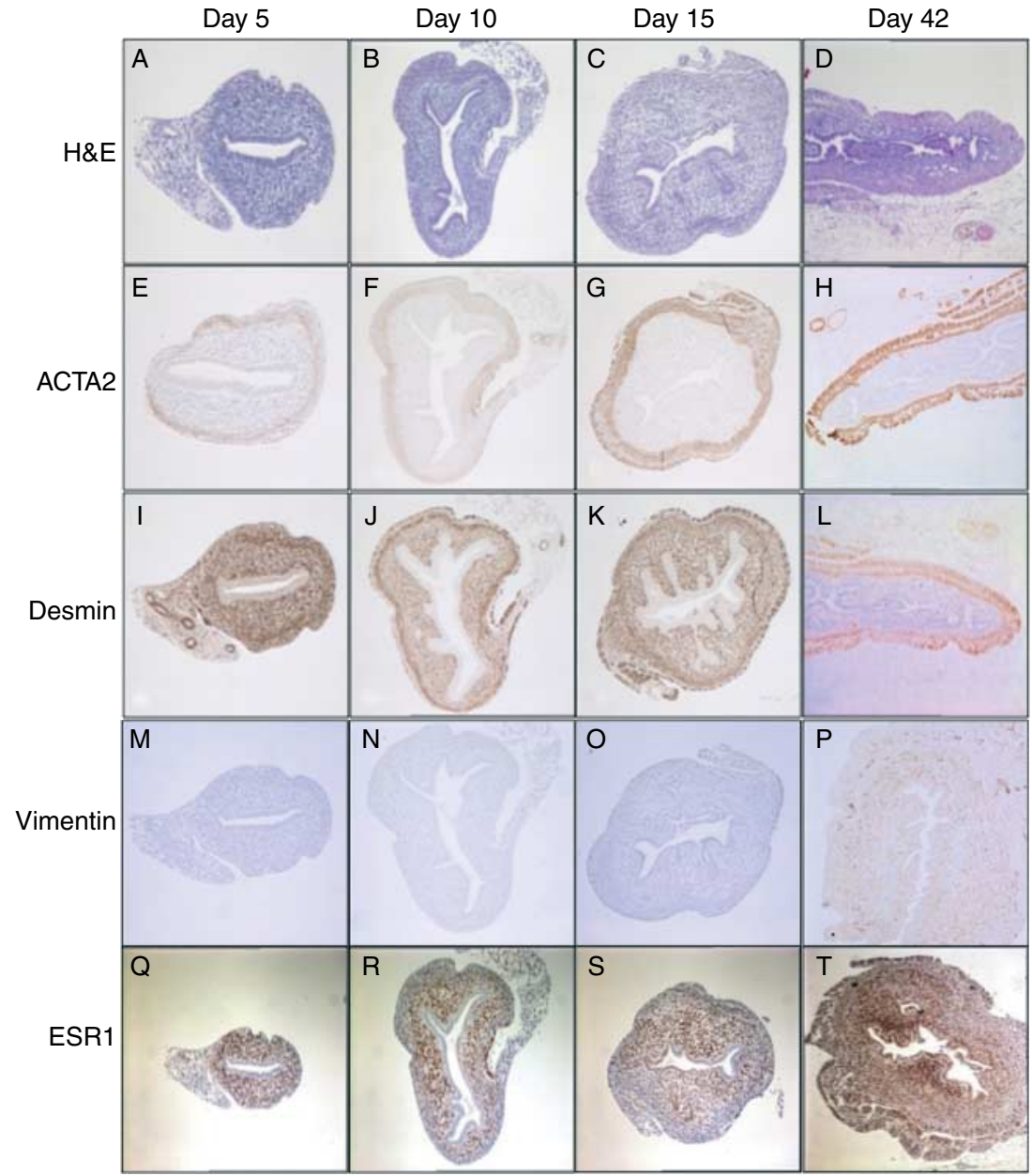

Figure 1 Control C57/BL6] mice. Haematoxylin and eosin (A-D), and immunohistochemical staining patterns for $\alpha$-smooth muscle actin $(\mathrm{E}-\mathrm{H})$, desmin $(\mathrm{I}-\mathrm{L})$, vimentin $(\mathrm{M}-\mathrm{P})$ and ESR1 (Q-T). Magnification: days 5, 10 and 15 micrographs $(\times 20)$, and day $42(\times 10)$. 


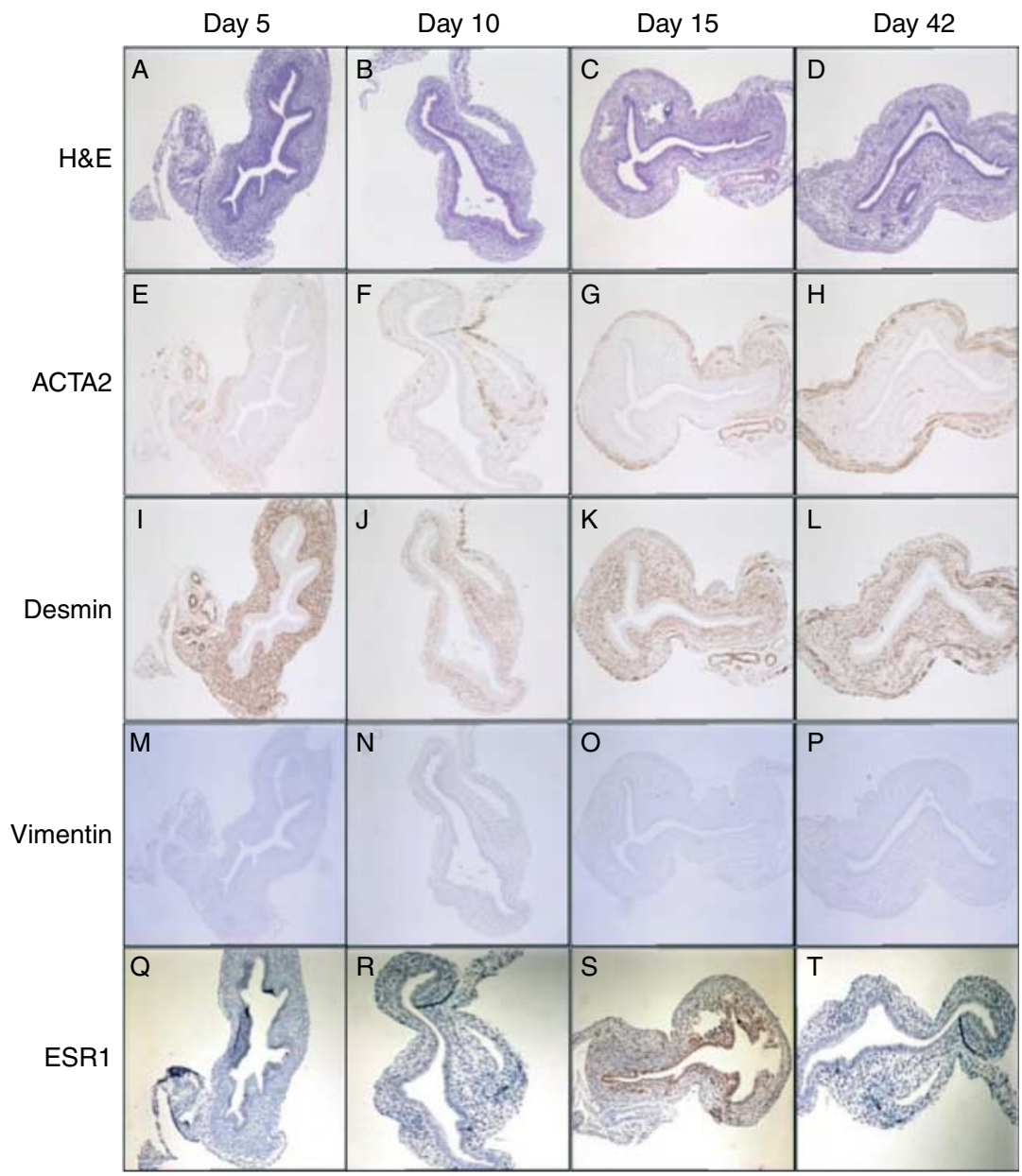

Figure 2 Tamoxifen-treated C57/BL6J mice. Haematoxylin and eosin (A-D), and immunohistochemical staining patterns for $\alpha$-smooth muscle actin $(\mathrm{E}-\mathrm{H})$, desmin $(\mathrm{I}-\mathrm{L})$, vimentin (M-P) and ESR1 (Q-T). Magnification: days 5, 10 and 15 micrographs $(\times 20)$, and day $42(\times 10)$. vessels. The outer longitudinal muscle layer was composed of prominent bundles of cells with wellformed connective tissue sheaths (Figs 1D-5D).

\section{Uterine development following tamoxifen administration}

Day 5. The uterine epithelium was taller, with large pale elongated nuclei and prominent nucleoli (Table 1). The uterine lumen was markedly dilated with prominent secretions, and the uteri were significantly heavier than controls. Blood-filled spaces were seen throughout the uterine wall, which was thinner compared to controls. Some cells in the middle of the uterine wall appeared to adopt circular orientation, but these were loosely bundled and only formed a thin patchy ring encircling about $25-40 \%$ of the uterus (Figs $2 \mathrm{~A}-5 \mathrm{I}$ ).

Day 10. The uterine lumen was dilated, and the endometrial stroma was highly cellular with ovoid nuclei. The luminal and glandular epithelia remained increased in height compared to controls. The myometrial layers were not distinct. Some cells had circular orientation but with no observable continuity. The interrupted bundles were surrounded by cellular stroma that appeared continuous with the endometrial stroma. The outer muscle layer was less obvious, with some bundles observed on the periphery (Figs 2B-5J).

Day 15 . The luminal and glandular epithelia were higher than controls. The circular muscle layer cells acquired darker staining cytoplasm and more obvious circular orientation. The outer myometrium showed signs of differentiation into bundles surrounded by thin loose connective tissue. The intervening vascular layer was not obvious, although some prominent blood vessels were seen scattered in the uterine wall (Figs $2 \mathrm{C}-5 \mathrm{~K}$ ).

Day 42. The uteri were smaller compared to controls. The endometrial stroma was highly cellular, and the glands developed normally, with the lining epithelium remaining higher than controls. The circular myometrium showed marked loosening and increased intercellular space. The outer myometrium was well developed with obvious bundling. Overall, the muscle integrity was more preserved on the anti-mesometrial border of the uterus. In summary, although the overall structure of the uterus was maintained and the outer longitudinal muscle layer developed normally, the development of the inner circular muscle layer was impaired, with marked dispersal of these cells. Overall, 


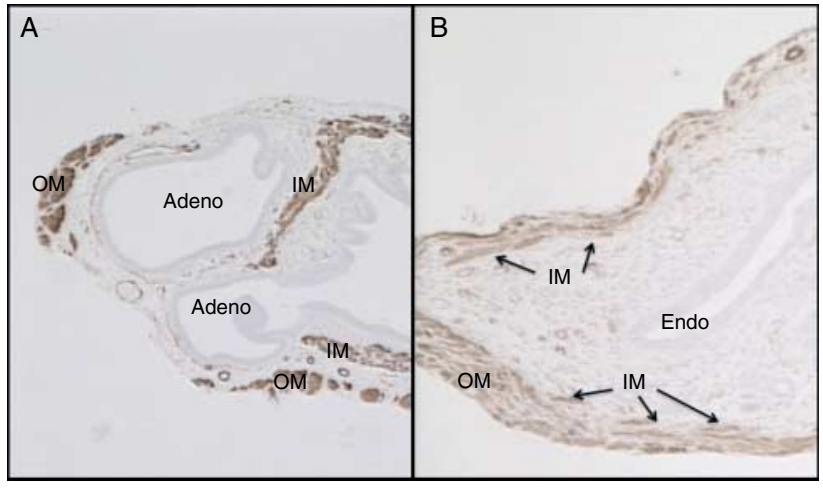

Figure 3 Comparison between day 42 uteri of (A) CD-1 mice treated with tamoxifen and (B) C57/BL6J mice treated with tamoxifen. The inner myometrium (IM) is disrupted in both species, with the outer myometrium (OM) formed normally. The endometrium (Endo) is well contained in the C57/BL6J uterus, but invades deeper to the inner myometrium in the CD-1 uterus, forming adenomyosis foci (Adeno). Some adenomyosis foci are subserosal, and there is attenuation of the vascular layer in both uteri. Magnification: $(\times 10)$.

the muscle integrity was more preserved on the antimesometrial border of the uterus. Adenomyosis was not detected at any stage (Figs 2D, 3 and $5 \mathrm{~L}$ ).

\section{Immunohistochemical markers}

\section{$\alpha$-Smooth muscle actin expression}

A narrow but distinct zone of variable staining intensity corresponding to the developing inner circular myometrium was seen in the middle of the uterine wall by day 5 . On day 10 , this layer strongly expressed $\alpha$-smooth muscle actin (ACTA2). Expression in the outer longitudinal muscle layer was less intense. Uteri from day 15 onwards exhibited well-defined, intense staining of both the inner and outer muscle layers (Figs $1 \mathrm{E}-\mathrm{H}$ and $5 \mathrm{E}-\mathrm{H}$ ).

In the tamoxifen group, ACTA2 expression was weaker, with fewer positive cells per area, reflecting disruption and loosening of the circular myometrium. Staining intensity was generally weaker in the inner circular as compared to the outer longitudinal muscle layers (Figs $2 \mathrm{E}-\mathrm{H}$ and $5 \mathrm{M}-\mathrm{P}$ ).

\section{Desmin expression}

In control C57/BL6J mice, desmin expression followed a 'wave' of maturation from inside outwards. Initially, on day 5, the developing endometrial stroma strongly expressed desmin, while the myometrial uterine wall remained less intense. By days 10 and 15, as the inner and outer and longitudinal myometrium layers developed, they acquired and expressed stronger immunostaining to desmin compared to the endometrial stroma that reverted to be weakly positive. The blood vessel walls stained strongly positive, while endometrial epithelium was negative (Fig. 1I-L). In pups receiving tamoxifen, this 'wave' of maturation was absent. The intensity of staining remained uniformly weak throughout the developmental phases (days 5-42) of the uterine wall structures. Generally, the intensity of staining was weaker than in controls. The endometrial epithelium remained negative (Fig. 2I-L).

\section{Vimentin expression}

Vimentin expression was observed in the endometrial stroma and blood vessel walls on days 15 and 42, in both groups. No staining was seen in the myometrium. In some uteri, occasional staining was observed in the developing stroma on day 5, but this was not consistent. Blood vessel walls stained positive and were considered intrinsic quality control (Figs 1, 2M-P and 4).

\section{Laminin and fibronectin expression}

On day 5, laminin expression was more localised to the middle third of the developing uterus, i.e. the prospective inner circular muscle layer, while fibronectin was differentially expressed in the prospective endometrial stroma or the inner third of the developing uterus. The same expression pattern was observed on day 10 and subsequently. Laminin was exclusively expressed in the extracellular matrix surrounding the smooth muscle layers (inner circular and outer longitudinal). Fibronectin was exclusively expressed around the stromal cells and in the connective tissue sheaths separating the outer longitudinal muscle, and the intervening vascular layer. There was no difference in the localisation of laminin or

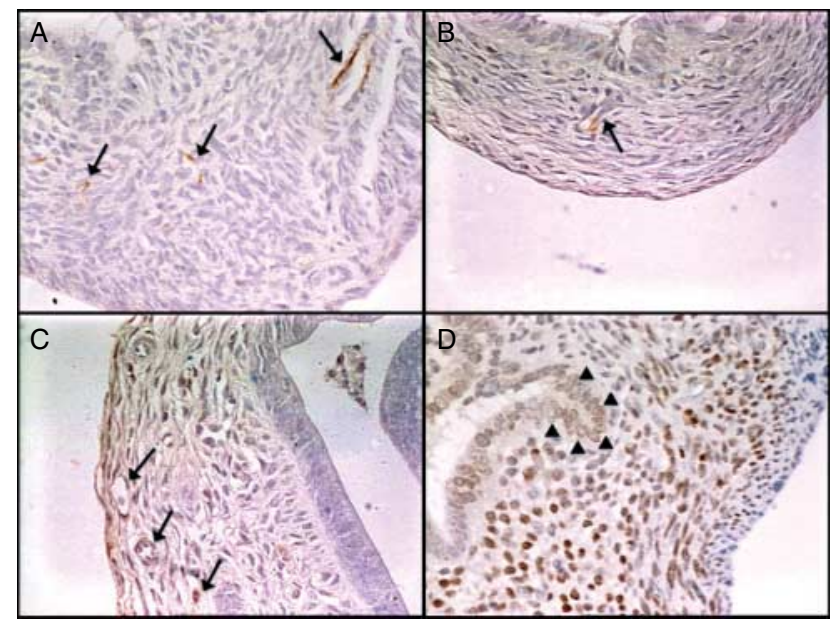

Figure 4 Immunohistochemical staining of (A) vimentin, day 15 , control C57/BL6J uterus; (B) vimentin, day 15, tamoxifen-treated C57/BL6J uterus; and (C) vimentin, day 42, tamoxifen-treated C57/BL6J uterus. Vimentin expression was observed in the endometrial stroma and blood vessel walls (arrows). No staining was seen in the myocytes. (D) ESR1 immunostaining, day 15, control uterus showing staining in the developing neck glands (arrow heads). Magnification: $(\times 40)$. 


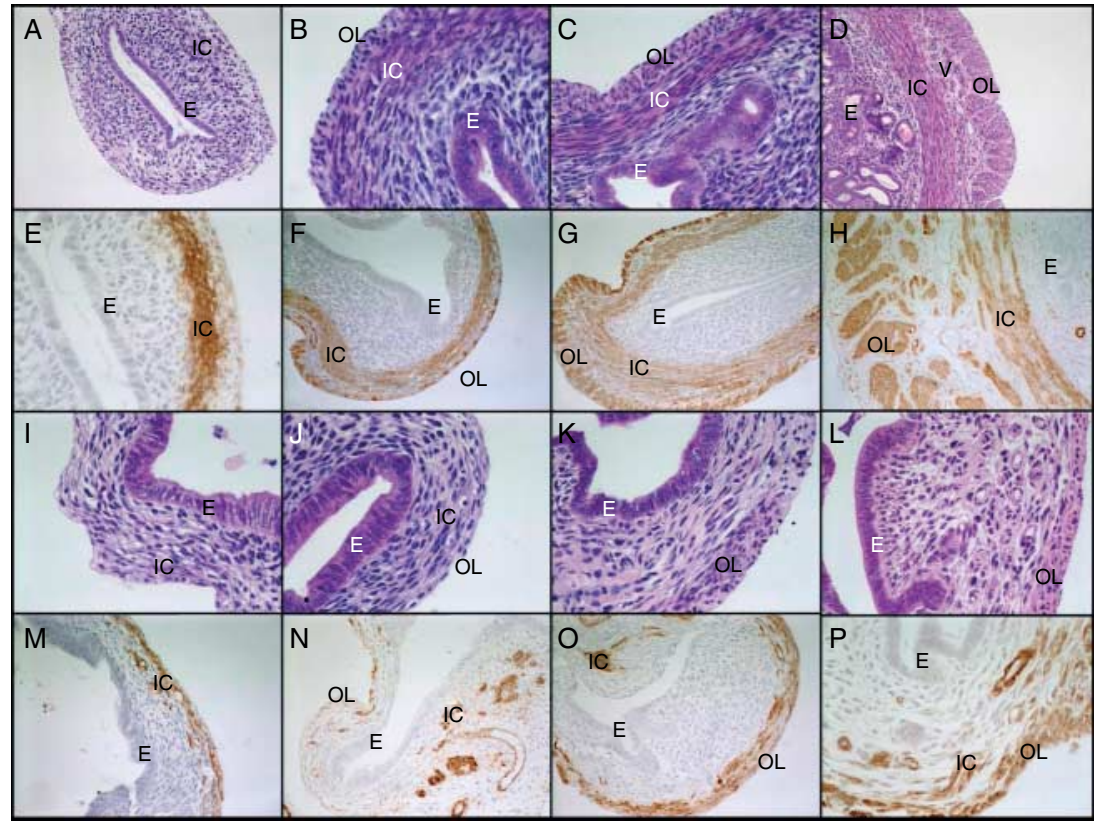

Figure 5 (A-D) Haematoxyin- and eosin-stained section of control C57/BL6J mice (days 5, 10, 15 and 42). Mesenchymal cells appear to segregate into three layers: endometrium and endometrial stroma (E), inner circular (IC) and outer longitudinal $(\mathrm{OL})$ muscle layers. A distinct loose vascular layer (V) separated the inner and outer myometrial layers from day 15 onwards. (E-H) (days 5, 10, 15 and 42) ACTA2 immunostaining showing the progressive development of the IC and OL muscle layers. (I-L) Haematoxylin and eosin stained and (M-P) (days 5, 10, 15 and 42) ACTA2 immunostained uterine sections from tamoxifen-treated mice. Note the dilated uterine lumen, prominent blood vessels and thinner uterine walls. The IC muscle layer is poorly developed and fragmented. The myometrial layers are less distinct, and although the overall structure of the uterus was maintained and the OL muscle layer developed normally, the development of the IC muscle layer was impaired, with marked dispersal of these cells. Adenomyosis was not detected at any stage.

fibronectin between the controls or tamoxifen groups. However, laminin expression was reduced in the tamoxifen group.

\section{Oestrogen receptor- $\alpha$ expression}

In the control mice, the whole thickness of the uterine wall expressed oestrogen receptor- $\alpha$ (ESR1) from day 2, while the epithelial lining remained negative till day 10 when the glandular epithelium in the neck glands exhibited some staining. As the various layers developed in the mesenchyme, ESR1 immunostaining was more intense in the stroma as compared to the muscle layers. Staining intensity was more variable by day 42 . This variation may or may not represent a reflection of oestrous cycle-related changes. This cannot be ascertained, as we did not perform any vaginal smears (Figs 1Q-T and 4).

In tamoxifen-treated mice, the overall ESR1 expression was attenuated compared to control uteri. There was marked reduction of ESR1 immunostaining, with no or very weak staining in all layers on days 5 and 10 . However, by day 15, the ESR1 staining has recovered in the endometrial layers (luminal epithelium and stroma), but was less obvious in the myometrium. On day 42, the glands and stroma were less positive, and both inner and outer muscle layers remained faintly immunopositive (Fig. 2Q-T).

\section{Discussion}

Perinatal uterine development represents a critical phase for uterine morphogenesis and differentiation (Iguchi \& Sato 2000). In a previous study, we demonstrated that neonatal exposure to tamoxifen in CD-1 mice, but not $\mathrm{OE}_{2}$, resulted in failure of full differentiation and development of the inner circular myometrial layer (Mehasseb et al. 2009). In CD-1 mice receiving tamoxifen, some cells in the middle of the uterine wall appeared to acquire a circular orientation on day 5 , but these were loosely bundled and only formed a thin patchy ring encircling about a third of the uterus. By day 10 , the muscle layers were not distinct. Some cells had a circular orientation, but there was no continuity and glands were seen deeper to the circular layer. The outer muscle layer was less obvious, with some bundles observed on the periphery. On day 15, the circular muscle layer disruption was more evident, and glands were seen extending deeper into the circular muscle layer. The outer myometrium showed signs of differentiation into bundles surrounded by thin loose connective tissue. Adenomyosis was first noted on day 5, and was seen in most specimens on day 15 . Ingrowth of endometrial glands and stroma was observed deep within the myometrium representing premature development of adenomyosis (Mehasseb et al. 2009). In the present study, we report that in C57/BL6J mice, 
which are constitutionally smaller than CD-1 mice but in which uterine developmental stages are similar (Cunha 1976, Brody \& Cunha 1989, Cunha et al. 1992), exposure to tamoxifen also resulted in disruption of uterine myometrial development, but unlike in CD-1 mice, it did not result in the ingrowth of the endometrium and development of adenomyotic changes. The similar effects upon myometrial development include that upon ACTA2, desmin and vimentin expression in the inner myometrial and of extracellular matrix components. The effect upon the circular inner myometrial layer in the C57/BL6J mouse was maintained into the adult period without any indication of the development of adenomyosis.

Tamoxifen affected the morphological development of the inner circular muscle layer where normally muscle cells would be closely packed in circular orientation. Additionally, there was some evidence that it was associated with a failure of full visceral smooth muscle cell differentiation. Thus, although developing myometrium expressed some immunohistochemical markers indicative of myogenic differentiation such as ACTA2, expression of the latter was delayed and attenuated, and cells exhibited reduced desmin expression. This may indicate that tamoxifen exposure during this critical neonatal period may inhibit full differentiation of mesenchymal cells to smooth muscle cells, and their phenotype may resemble a form of intermediate myofibroblasts or immature smooth muscle cells. Reduced desmin in uterine muscle of tamoxifen-administered mice might also indicate a perturbation or dysfunction of their myofibres, leading to a reduction in tensile strength and disruption with the resultant abnormal architecture. Laminin is an important component of the basal lamina associated with smooth muscle and its expression being unaffected by tamoxifen may indicate normal smooth muscle differentiation; however, its expression is also associated with myofibroblasts and immature forms of smooth muscle cells.

Neonatal exposure to tamoxifen therefore interferes with subsequent uterine myometrial development, specifically the inner circular muscle coat. This does not appear to be strain specific. As tamoxifen effects are most likely to be mediated via ESRs, it is interesting to note that they have been identified in the epithelium, stroma and the myometrium of the developing uterus (Kurita et al. 2001). However, some strain-dependent differences in expression in the uterus of neonatal mice have been reported. In CD-1 mice, ER immunoreactivity was observed in stromal cells on day 1 and in epithelial cells on day 5 (Yamashita et al. 1989, Sato et al. 1992). In BALB/C mice, ER expression was delayed till day 5 in the stroma and until day 14 in the epithelial cells (Yamashita et al. 1989, Li 1994). Glandular development in the uteri of CD-1 mice has also been reported to be advanced by 3 days compared to BALB/c mice (Bigsby et al. 1990). In the absence of differences in the expression of ESR1 distribution in C57BL/6J and CD-1 strains, it is unlikely that the different responses to tamoxifen are attributed to differences in ESR1 expression in the myometrium.

Tamoxifen significantly downregulates ESR1 in C57/BL6J mice. Tamoxifen is able to antagonise the action of calmodulin and protein kinase $\mathrm{C}$, and compete for membrane-binding sites for neurotransmitter substances (Lam 1984, Kroeger \& Brandes 1985, Horgan et al. 1986, Batra 1990). Muscle strips from nonpregnant human myometrium showed marked inhibition of spontaneous and vasopressin-induced contractions when tamoxifen was added to the bathing solution (Kostrzewska et al. 1997). This spasmolytic activity was also demonstrated in the rat myometrium, where tamoxifen antagonised the contractions induced by exogenous calcium (Lipton \& Morris 1986). It is thus plausible that the myometrial abnormalities observed following tamoxifen administration could be related to dysfunction in the processes involved in the contractile machinery and the regulation of calcium metabolism.

Abnormal muscle development has previously been reported in experimentally induced adenomyosis. In prolactin-induced adenomyosis, the myometrium becomes loose with increased intercellular space, and muscle cells become small and irregular. Disintegration of the muscle layer, with reduced cell organelles, and pyknosis of the myocytes were frequently seen in areas of adenomyosis (Mori et al. 1984, Ohta et al. 1985). The fact that in C57/BL6J mouse strain tamoxifen-induced changes were not associated with adenomyosis per se suggests an additional factor is required. It may be of relevance that neonatal tamoxifen exposure caused early adenomyosis only in the CD-1 strain, which exhibits a high rate of spontaneous adenomyosis during late adult life. The presence of inner myometrial defects caused by tamoxifen may allow the constitutively 'invasive' endometrium to invade the inner muscle coat resulting in premature adenomyosis. This would suggest that in the 'predisposed' CD-1 strain, the glandular and/or stromal components of the endometrium possess a higher invasive capacity. Thus, in the C57/BL6J strain, although tamoxifen produces the same circular muscle coat defect, the endometrium does not invade the disrupted muscle layer and does not present with adenomyosis. It is interesting to note that in the SHN strain, which also exhibits age-related spontaneous adenomyosis, the administration of the matrix metalloproteinase inhibitor ONO-4817 retarded the progression of uterine adenomyosis (Mori et al. 2002), which again suggests a role for an aggressive stromal invasion. The absence of differences in the extracellular matrix proteins laminin and fibronectin following tamoxifen administration (Romberger 1997, Sasaki et al. 2004) also suggests that alterations in the myometrial layers are the primary causes of endometrial invasion into these tissues.

A strong correlation between the incidence of uterine adenomyosis and of mammary tumours in the SHN 
mouse, a strain in which there is a high incidence of spontaneous tumour development, has been reported (Mori et al. 2002). Additionally, concomitant development of uterine and breast lesions has also been observed in SLN, GR/A and C3H/He strains (Nagasawa et al. 1987, Nagasawa \& Kusakawa 2001). Nothing has been reported regarding the neonatal effects of oestrogens. Therefore, it would be interesting to determine whether a similar correlation exists between strains that exhibit tamoxifen-induced adenomyosis and the incidence of spontaneous mammary lesions and tumours in these strains. Medroxyprogesterone acetate increased the incidence of both uterine and mammary lesions in SHN mice, whereas RU486 stimulated mammary tumours but inhibited uterine adenomyosis (Nagasawa et al. 1989). Disruption of development of the inner myometrium cannot explain the development of uterine adenomyosis, and although these changes may be a prerequisite for the premature development of adenomyosis, additional genetic or epigenetic differences between strains expressed in the endometrium may be required. Elucidation of these strain-dependent mechanisms may contribute to our understanding of the aetiopathology of adenomyosis in the humans.

In conclusion, our results demonstrate that the development of the inner myometrium is particularly sensitive to oestrogen antagonism, and is affected by steroid receptor modulation and is not due to the strain-dependent factors.

\section{Materials and Methods}

The study was conducted under the authority of the United Kingdom Home Office, Animals (Scientific Procedures) Act 1986. Pregnant time-mated C57BL/6J mice were obtained (Charles River Ltd, Margate, Kent, UK), and their female pups were divided into two groups. The first group $(n=20)$ was orally dosed on days 1-5 after birth (day of birth being d0) with $1 \mathrm{mg} / \mathrm{kg}$ tamoxifen suspended in peanut oil/lecithin/condensed milk mixture at a concentration of $200 \mu \mathrm{g} / \mathrm{ml}$, and at a dose of $5 \mu \mathrm{l} / \mathrm{g}$ body weight. The second control group received vehicle only. Mice from the study group $(n=5)$ and from the control group were culled on days 5, 10, 15 and 42. Uteri were collected, weighed and fixed in $4 \%$ neutral buffered formalin for $24 \mathrm{~h}$ at room temperature. Paraffin-embedded cross sections ( $2 \mu \mathrm{m}$ thin to avoid overlap of cells) were cut and mounted on silane-coated glass slides for histological and immunohistochemical examination.

\section{Immunohistochemistry}

Sections were dewaxed in xylene, and rehydrated in graded alcohols and water. Epitope antigen retrieval using microwave and citrate buffer $(\mathrm{pH}=6.0)$ was used for desmin, fibronectin and ESR1 staining. Endogenous peroxidase activity was blocked with $6 \%(\mathrm{v} / \mathrm{v})$ hydrogen peroxide $\left(\mathrm{H}_{2} \mathrm{O}_{2}\right)$ in water for $10 \mathrm{~min}$. Specific mouse-on-mouse blocking reagent
(Vector Labs, Peterborough, UK) was applied for $1 \mathrm{~h}$, where applicable. Sections were incubated overnight at $4{ }^{\circ} \mathrm{C}$ with the primary antibodies against desmin (mouse monoclonal clone DR33, 1:50 (v/v), Dako, Cambridge, UK), vimentin (mouse monoclonal, clone LN6, 1:50 (v/v), Chemicon, Millipore, Watford, UK), laminin (rabbit polyclonal, 1:200 (v/v), Abcam, Cambridge, UK), fibronectin (rabbit polyclonal, 1:500 (v/v), Abcam), and ESR1 (mouse monoclonal, clone ER-6F11, Novocastra, Newcastle upon Tyne, UK), and for $1 \mathrm{~h}$ at room temperature for ACTA2 (clone SMM1, Vector Labs). Biotinylated rabbit anti-mouse or swine anti-rabbit secondary antibody (Dako) was applied at a concentration of 1:400 (v/v) for $30 \mathrm{~min}$ at room temperature. Immunoreactivity was demonstrated with 3,3'-diaminobenzidine $/ \mathrm{H}_{2} \mathrm{O}_{2}$ (DAB solution; Vector Labs). Sections were lightly counterstained with haematoxylin, then dehydrated and cleared in graded alcohol and xylene, and finally covered with glass slips. The immunostaining was assessed for distribution and intensity using a visual score $(-$, negative; \pm , equivocal; + , weak; ++ , moderate; +++ , strong). Antibodies used for this study have been previously tested and validated for specificity in similar tissue sources as used here (Mehasseb et al. 2009). Image capture and analysis were performed using Axioplan 2 light microscopy (Carl Zeiss, Gottingen, Germany) and an image capture system. The system was based on a single chip colour video camera (Sony DXC-151P; Sony Inc., Tokyo, Japan) connected to a camera adapter (Sony CMA-151P, Sony Inc.) that transmits the image to a Windows-based computer via a Meteor 2 MMC graphics display interface and the Axiovision image analysis software (version 4.0, Carl Zeiss).

\section{Declaration of interest}

The authors declare that there is no conflict of interest that could be perceived as prejudicing the impartiality of the research reported.

\section{Funding}

This work was supported by the University of Leicester and the University Hospitals of Leicester NHS Trust.

\section{Acknowledgements}

We would like to thank Dr lan White (Honorary Reader, University of Leicester, UK) for his advice. We would also like to thank the Staff at the Division of Biomedical Services, University of Leicester for technical assistance.

\section{References}

Batra S 1990 Interaction of antiestrogens with binding sites for muscarinic cholinergic drugs and calcium channel blockers in cell membranes. Cancer Chemotherapy and Pharmacology 26 310-312.

Bigsby RM, Aixin L, Luo K \& Cunha GR 1990 Strain differences in the ontogeny of estrogen receptors in murine uterine epithelium. Endocrinology 126 2592-2596.

Bird CC, McElin TW \& Manalo-Estrella P 1972 The elusive adenomyosis of the uterus - revisited. American Journal of Obstetrics and Gynecology $112583-593$. 
Brody JR \& Cunha GR 1989 Histologic, morphometric, and immunocytochemical analysis of myometrial development in rats and mice. I. Normal development. American Journal of Anatomy 186 1-20.

Cunha GR 1976 Stromal induction and specification of morphogenesis and cytodifferentiation of the epithelia of the mullerian ducts and urogenital sinus during development of the uterus and vagina in mice. Journal of Experimental Zoology 196 361-370.

Cunha GR, Battle E, Young P, Brody J, Donjacour A, Hayashi N \& Kinbara H 1992 Role of epithelial-mesenchymal interactions in the differentiation and spatial organization of visceral smooth muscle. Epithelial Cell Biology 1 76-83.

Curtis KM, Hillis SD, Marchbanks PA \& Peterson HB 2002 Disruption of the endometrial-myometrial border during pregnancy as a risk factor for adenomyosis. American Journal of Obstetrics and Gynecology 187 543-544.

Greaves P \& White IN 2006 Experimental adenomyosis. Best Practice \& Research. Clinical Obstetrics \& Gynaecology 20 503-510.

Green AR, Styles JA, Parrott EL, Gray D, Edwards RE, Smith AG, Gant TW, Greaves P, Al-Azzawi F \& White IN 2005 Neonatal tamoxifen treatment of mice leads to adenomyosis but not uterine cancer. Experimental and Toxicologic Pathology 56 255-263.

Guttner J 1980 Adenomyosis in mice. Zeitschrift für Versuchstierkunde 22 249-251.

Horgan K, Cooke E, Hallet MB \& Mansel RE 1986 Inhibition of protein kinase $\mathrm{C}$ mediated signal transduction by tamoxifen. Biochemical Pharmacology 35 4463-4465.

Huseby RA \& Thurlow S 1982 Effects of prenatal exposure of mice to "lowdose" diethylstilbestrol and the development of adenomyosis associated with evidence of hyperprolactinemia. American Journal of Obstetrics and Gynecology 144 939-949.

Iguchi T \& Sato T 2000 Endocrine disruption and developmental abnormalities of female reproduction. American Zoologist 40 402-411.

Irisawa S \& Iguchi T 1990 Critical period of induction by tamoxifen of genital organ abnormalities in female mice. In Vivo 4 175-179.

Kostrzewska A, Laudanski T \& Batra S 1997 Potent inhibition by tamoxifen of spontaneous and agonist-induced contractions of the human myometrium and intramyometrial arteries. American Journal of Obstetrics and Gynecology 176 381-386.

Kroeger EA \& Brandes LJ 1985 Evidence tat tamoxifen is a histamine antagonist. Biochemical and Biophysical Research Communications 131 750-755.

Kurita T, Cooke PS \& Cunha GR 2001 Epithelial-stromal tissue interaction in paramesonephric (Mullerian) epithelial differentiation. Developmental Biology 240 194-211.

Lam HY 1984 Tamoxifen is a calmodulin antagonist in the activation of cAMP phosphodiesterase. Biochemical and Biophysical Research Communications 118 27-32.

Levgur M, Abadi MA \& Tucker A 2000 Adenomyosis: symptoms, histology, and pregnancy terminations. Obstetrics and Gynecology 95 688-691.

Li S 1994 Relationship between cellular DNA synthesis. PCNA expression and sex steroid hormone receptor status in the developing mouse ovary, uterus and oviduct. Histochemistry 102 405-413.

Lipton A \& Morris ID 1986 Calcium antagonism by the antioestrogen tamoxifen. Cancer Chemotherapy and Pharmacology 18 17-20.

McCann TO \& Myers RE 1970 Endometriosis in rhesus monkeys. American Journal of Obstetrics and Gynecology 106 516-523.

Mehasseb MK, Bell SC \& Habiba MA 2009 The effects of tamoxifen and estradiol on myometrial differentiation and organization during early uterine development in the CD1 mouse. Reproduction 138 341-350.
Mori T \& Nagasawa H 1983 Mechanisms of development of prolactininduced adenomyosis in mice. Acta Anatomica 116 46-54.

Mori T, Nagasawa H \& Nakajima Y 1982 Strain-difference in the induction of adenomyosis by intrauterine pituitary grafting in mice. Laboratory Animal Science 32 40-41.

Mori T, Ohta Y \& Nagasawa H 1984 Ultrastructural changes in uterine myometrium of mice with experimentally-induced adenomyosis. Experientia 40 1385-1387.

Mori T, Singtripop T \& Kawashima S 1991 Animal model of uterine adenomyosis: is prolactin a potent inducer of adenomyosis in mice? American Journal of Obstetrics and Gynecology 165 232-234.

Mori T, Nakahashi K, Kyokuwa M, Yamasaki S \& Nagasawa H 2002 A matrix metalloproteinase inhibitor, ONO-4817, retards the development of mammary tumor and the progression of uterine adenomyosis in mice. Anticancer Research 22 3985-3988.

Nagasawa H \& Kusakawa S 2001 Relationship between incidence and onset age of mammary tumours and uterine adenomyosis in four strains of mice: comparison with the findings of 40 generations previously. In Vivo 15 345-349.

Nagasawa H, Konishi R, Naito T, Ohmiya S \& Mori T 1987 Relationship between mammary tumourigenesis and uterine adenomyosis in four strains of mice. In Vivo 1 237-240.

Nagasawa H, Aoki M, Mori T, Yamamoto K, Inaba T \& Mori J 1989 Stimulation of mammary tumourigenesis and inhibition of uterine adenomyosis by suppressed progesterone effects in SHN mice. Anticancer Research 9 827-832.

Ohta Y, Mori T \& Nagasawa H 1985 Ultrastructural changes of endometrium and myometrium during development of adenomyosis in mice with ectopic pituitary transplants. Zoological Science 2 239-247.

Ostrander PL, Mills KT \& Bern HA 1985 Long-term responses of the mouse uterus to neonatal diethylstilbestrol treatment and to later sex hormone exposure. Journal of the National Cancer Institute 74 121-135.

Parrott E, Butterworth M, Green A, White IN \& Greaves P 2001 Adenomyosis - a result of disordered stromal differentiation. American Journal of Pathology 159 623-630.

Romberger DJ 1997 Fibronectin. International Journal of Biochemistry and Cell Biology 29 939-943.

Sasaki T, Fassler R \& Hohenester E 2004 Laminin: the crux of basement membranes assembly. Journal of Cell Biology $164959-963$.

Sato T, Okamura H, Ohta Y, Hayashi S, Takasugi N \& Iguchi T 1992 Estrogen receptor expressin in genital tract of female mice treated neonatally with diethylstilbestrol. In Vivo 6 151-156.

Suire RA, Goodman DG, Valerio MG, Fredrickson TN, Strandberg JD, Levitt MH, Lingeman CH, Harshbarger JC \& Dawe CJ 1978 Female reproductive system. In Pathology of Laboratory Animals, vol 2, pp 1051-1262. Eds K Benirschke, FM Garner \& TC Jones. New York: Springer-Verlag.

Yamashita S, Newbold RR, McLachlan JA \& Korach KS 1989 Developmental pattern of estrogen receptor expression in female mouse genital tracts. Endocrinology 125 2888-2896.

Received 7 October 2009

First decision 6 November 2009

Revised manuscript received 4 February 2010

Accepted 16 March 2010 